ATLAS Internal Note

INDET-No-123

March 29, 1996

\title{
Spatial Resolution Measurements of Gallium Arsenide Microstrip Detectors
}

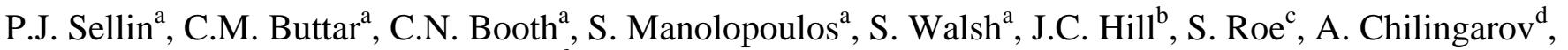 \\ F. Fares ${ }^{\mathrm{e}}$, G.F. Moorhead ${ }^{\mathrm{e}}$, S. Stapnes ${ }^{\mathrm{f}}$ and W. Murray ${ }^{\mathrm{g}}$ \\ ${ }^{a}$ Department of Physics, The University of Sheffield \\ ${ }^{b}$ Cavendish Laboratory, The University of Cambridge \\ ${ }^{c}$ European Laboratory for Particle Physics (CERN), Geneva \\ ${ }^{d}$ Department of Physics, University of Lancaster \\ ${ }^{e}$ Research Centre for High Energy Physics, The University of Melbourne \\ ${ }^{f}$ The University of Oslo \\ ${ }^{g}$ Rutherford Appleton Laboratory, Didcot
}

Presented by P.J. Sellin at the 1995 Nuclear Science Symposium, San Francisco, and submitted to Nuclear Instruments and Methods in Physics Research.

\begin{abstract}
Results are reported from beam test measurements of one of the first commercial prototype gallium arsenide microstrip detectors. The detector consisted of 128 strips on a $50 \mu \mathrm{m}$ pitch, with a wafer thickness of $200 \mu \mathrm{m}$. Operating at a bias of $180 \mathrm{~V}$, the detector produced a signal to noise ratio of 19 to 1 , with a particle detection efficiency of better than $99 \%$. The spatial resolution of the detector was measured as $14.6 \mu \mathrm{m}$ without charge sharing effects. Charge sharing between pairs of strips was seen to improve the spatial resolution, with an average value for all events of $10.4 \mu \mathrm{m}$.
\end{abstract}

\section{Introduction}

Recently the development of gallium arsenide (GaAs) microstrip detectors has been actively pursued for use as radiation hard tracking detectors for ATLAS at the Large Hadron Collider (LHC) at CERN [1]. Such devices are very similar in their design and operation to the more usual silicon microstrip detectors, but have some unique characteristics due to the inherent properties of GaAs. Recent activity to develop GaAs microstrip detectors has concentrated on the use of semi-insulating (SI) GaAs which is a compensated semiconductor with a high resistivity (typically $10^{7} \Omega \mathrm{cm}$ ) and which is readily available in wafers of suitable thickness (typically 200-500 $\mu \mathrm{m}$ ). However, due to the presence of charge traps in this material, particularly the EL2 trap at mid band gap, such devices have a charge collection efficiency which is less than $100 \%$ and which decreases with increasing wafer thickness. A high operating bias is also required (typically $1 \mathrm{~V}$ per micron of detector thickness) for the electric field to penetrate fully through the detector and so maximise the collected charge for minimum ionising particles (MIPs). Many recent papers have been published which summarise the charge collection properties of SI GaAs detectors; a comprehensive recent review is provided in reference [2].

The GaAs strip detectors studied in this work were some of the first commercially fabricated prototype devices, produced by EEV Ltd [3]. In this study, one of these detectors was exposed to a beam of charge 
particles in the $\mathrm{H} 8$ test-beam facility at CERN to study the spatial resolution of the device and the possible influence of charge trapping. The detector was instrumented using an analogue readout system, so that the pulse height from every strip was recorded on an event by event basis. This allowed charge sharing between strips and any corresponding effect on the detector resolution to be investigated.

\section{Experimental Apparatus}

\subsection{The GaAs microstrip detectors}

The GaAs detectors were produced by EEV Ltd, using a mask layout designed at the University of Sheffield. The detectors were fabricated from $200 \mu \mathrm{m}$ thick semi insulating GaAs, produced using the vertical gradient freeze method by AXT Ltd. The GaAs was supplied as three inch diameter wafers, with a nominal resistivity of $>1 \times 10^{7} \Omega \mathrm{cm}$. The detectors consisted of a patterned Ag/Ti Schottky contact on one side (the 'front' surface) and a planar AuGe/Ni ohmic contact on the reverse side (the 'rear' surface). Fabrication of the front surface used a three step mask process, with a silicon nitride passivation layer deposited during the second step.

The mask contained a variety of microstrip detector designs, plus a selection of pad detectors which were used for detector characterisation and for radiation damage studies. Results from these radiation damage studies have recently been published separately [4]. The strip detector consisted of 128 parallel strips on a $50 \mu \mathrm{m}$ pitch, with a strip width of $30 \mu \mathrm{m}$. Each strip was $8300 \mu \mathrm{m}$ long, and contained 3 bond pads at one end and two bond pads at the other end. A single guard ring surrounded the entire structure, with dimensions between the outside edges of the guard ring of $8.5 \mathrm{~mm}$ in length by $7 \mathrm{~mm}$ across. A $300 \mathrm{~nm}$ thick layer of silicon nitride was deposited over the strips to act both as a passivation layer and to provide an integrated decoupling capacitor. A similarly patterned upper gold layer was laid on top of the silicon nitride to form the upper capacitive contact.

Each detector strip was biased through the lower metal layer via an external resistor biasing network, which was connected to the detector using gold wire bonds. The resistor network [5] consisted of an array of resistive tracks on a ceramic substrate, formed from sputtered silicon-doped nichrome. Each array contained 384 resistive tracks approximately $12 \mathrm{~mm}$ in length and laid on a $50 \mu \mathrm{m}$ pitch, which were connected to a common bias bar at one end and contained a pair of bonding pads at the other end of each track. The resistance for each array was between $20 \mathrm{M} \Omega$ and $50 \mathrm{M} \Omega$, obtained with a precision better than $5 \%$ across any single array.

At the other end of each detector strip a bond wire was connected from a bond pad on the upper metal layer to an external capacitor array [6]. The external capacitor array was inserted between the readout electronics and the detector to provide an additional decoupling capacitor. This followed laboratory tests of the integrated silicon nitride decoupling capacitor which showed a significant number of short circuits through the silicon nitride layer due to damage after wire bonding.

The detector module consisting of the GaAs detector, the biasing network and the capacitor array were mounted onto a PCB which contained an aperture measuring approximately $7 \mathrm{~mm}$ by $8.5 \mathrm{~mm}$ underneath the detector. External connections for the biasing circuit were carried across the PCB on strips of kapton cable and connected using bond wires. The detector module was glued to the PCB containing the readout chip, which was then bonded to the decoupling capacitor. 


\subsection{Readout electronics}

Integration of the charge induced in each detector strip was performed by a single 128 channel VA2 low noise readout chip [7]. Each channel of the VA2 contains a charge sensitive preamplifier, a signal shaper, and a sample and hold circuit. The shaping time of the VA2 was set to $1.0 \mu$ s, so as to minimise the electronic noise on the output signal. The VA2 chip was mounted on a PCB hybrid containing the digital control lines, which were driven from an external NIM driver unit. The differential analogue output signals from the VA2 were digitised using a VME-mounted SIROCCO flash ADC [8]. The data read from the SIROCCO was combined by the data acquisition system with that from the silicon telescope and the other detectors under test, and written to tape.

\subsection{The test-beam facility}

These measurements were made at the ATLAS test-beam facility located in the H8 area at the CERN SPS, and were part of the wider programme for the development of ATLAS silicon strip detectors. First results from the prototype ATLAS silicon detectors have recently been published elsewhere [9]. The testbeam telescope consisted of $4 x y$ planes of silicon strip detectors, each plane made up of a pair of vertically and horizontally aligned single-sided silicon strip detectors. Each silicon detector contained 384 strips on a $50 \mu \mathrm{m}$ pitch, instrumented using VA2 electronics. The spatial resolution of the reconstructed telescope tracks was better than $2 \mu \mathrm{m}$. The primary trigger for the data acquisition system was defined by a pair of crossed scintillators immediately in front of the first silicon plane, with an overlapping area approximately $3 \mathrm{~cm}$ in height and $1 \mathrm{~cm}$ wide. The GaAs detector under test was inserted between the second and third silicon planes, orthogonal to the beam direction and with the strips arranged vertically.

\section{Signal to noise ratio and detector efficiency}

The charge collection efficiency of GaAs detectors has recently been extensively studied in the laboratory, using pad detectors irradiated with alpha particles and with minimum ionising electrons. The charge collection efficiency is observed to increase approximately linearly with increasing bias, corresponding to the penetration of a high electric field 'active' region extending from the blocking contact towards the rear surface of the detector. A description of the mechanisms affecting the charge collection response of these devices has been published recently [10].

The detectors supplied by EEV Ltd included both pad detectors and strip detectors fabricated from the same mask and from the same wafer. The charge collection efficiency of the pad detectors was measured in the laboratory using discrete readout electronics, for which an absolute charge calibration was possible. The charge collection efficiency was approximately 50\% (equivalent to approximately 13000 electrons for the most probably pulse height from MIPs) at a bias voltage of $180 \mathrm{~V}$. Although a higher charge collection efficiency has been observed for detectors fabricated from this material (65\% at 200V bias), breakdown prevented the strip detectors from running consistently at voltages above $180 \mathrm{~V}$. The operating temperature of the detectors in the test-beam was $28^{\circ} \mathrm{C}$ and the leakage current (including the guard ring) at $180 \mathrm{~V}$ was $3.9 \mu \mathrm{A}$.

The event by event data was recorded to tape and analysed off-line at CERN. The pulse height values from each strip were first corrected both for the pedestal of that channel and for common mode noise across the detector. The RMS noise in each strip was then calculated. Two groups of strips (strip numbers 44-50 and 88-93) were identified where the channel noise was several times greater than for the remaining 
strips, which was caused by faulty bond wires. These channels were masked out in the subsequent software analysis.

Particle hits in the detector were initially identified by searching for strips with a pulse height greater than five times the RMS noise (ie. $\mathrm{PH}>5 \sigma$ ). In addition searches were made for clusters of strips associated with a particle hit, where a cluster was defined as a number of adjacent strips where at least one strip contained a pulse height greater than $5 \sigma$ and the remaining strips had pulse heights which exceeded a defined minimum pulse height threshold. The optimum value for this threshold was determined within the range $0.5 \sigma$ to $5.0 \sigma$, as described in the following section.

For a given cluster pulse height threshold, the total pulse height $P H_{C L}$ for a cluster of $n$ strips is simply the sum of the pulse heights in the participating strips

$$
P H_{C L}=\sum_{i=1}^{n} P H_{i}
$$

and the cluster signal to noise ratio $S N_{C L}$ is given by

$$
S N_{C L}=\sum_{i=1}^{n} P H_{i} / \sigma_{i}
$$

Data was taken from the detector at four bias voltages from $120 \mathrm{~V}$ to $180 \mathrm{~V}$. A typical pulse height spectrum is shown in figure 1 , taken at a bias of $180 \mathrm{~V}$. The lack of events below channel 70 in this spectrum represents the software-imposed cluster threshold. Figure 2 shows the same data plotted in units of signal over noise where the most probable signal over noise value, calculated by fitting a gaussianconvoluted Landau distribution, is approximately 19.

Figure 3 shows a summary of the most probable values of the pulse height and signal to noise ratio which were obtained from several runs at four different detector bias voltages. As expected from the detector simulations, within this voltage range the most probable pulse height increases linearly with detector bias due to the increasing width of the active region of the detector. As detector breakdown prevented data from being taken at bias voltages above $180 \mathrm{~V}$, it was not possible to demonstrate the expected saturation of the charge collection efficiency at high bias.

The measured mean noise across all good channels only increased slightly with bias, from 8.5 channels RMS at $120 \mathrm{~V}$ to 9.2 channels RMS at $180 \mathrm{~V}$. Consequently the most probable signal to noise ratio increased approximately linearly over the voltage range (see figure $3 \mathrm{~b}$ ).

The hit efficiency of the GaAs strip detector was calculated from the proportion of particle tracks incident on the GaAs detector which generated valid detector hits of a either a single strip or a cluster of strips. Figure 4 shows the resulting hit efficiency distribution plotted as a function of signal/noise threshold. An efficiency of better than $99 \%$ was achieved for a signal/noise threshold of $5 \sigma$.

\section{Cluster size and spatial resolution}

In order to investigate the importance of charge sharing between strips and the resulting effect on the detector's spatial resolution, a study was made of the distribution of strip cluster widths at various bias voltages. As described previously, a cluster was defined in the off-line analysis program as a number of adjacent strips where at least one strip has a pulse height in excess of $5 \sigma$ and the remaining strips have 
pulse heights in excess of a precept threshold value. The optimum value of this cluster threshold was investigated over the range from $0.5 \sigma$ to $5.0 \sigma$, primarily by looking at the effect on the detector's spatial resolution. If the threshold is set too low the average number of strips per cluster will be artificially high, so degrading the detector's spatial resolution and increasing the risk of producing false hits. Conversely if the cluster threshold is set too high, the average number of strips per cluster will approach unity and any potential gain in spatial resolution due to valid charge sharing will be reduced.

The spatial resolution of the detector is defined in terms of the measured residual distribution, where for each event the residual $X_{R E S}$ is calculated within a local co-ordinate frame relative to the detector by

$$
X_{R E S}=X_{D E T}-X_{T E L}
$$

where $X_{D E T}$ is the position of the incident particle as defined by the GaAs detector and $X_{T E L}$ is the position of the particle track as reconstructed by the external silicon detector telescope. The spatial resolution of the GaAs detector $\left(\sigma_{D E T}\right)$ is given by

$$
\sigma_{D E T}^{2}=\sigma_{R E S}^{2}-\sigma_{T E L}^{2}
$$

where $\sigma_{R E S}$ is the RMS width of the residuals distribution and $\sigma_{T E L} \approx 2.0 \mu \mathrm{m}$.

\subsection{Spatial resolution without charge sharing}

Initially the spatial resolution of the detector was investigated with charge sharing effects excluded. This was achieved by defining valid particle hits solely by finding the single strip with the largest pulse height in each event, whilst still requiring a pulse height of $5 \sigma$ or greater. Since the flux of incident particles across any small area of the detector can be considered uniform, this analysis should produce a characteristic 'top hat' distribution for $X_{R E S}$, which has a width approaching the strip pitch of $50 \mu \mathrm{m}$ in the limit of $\sigma_{T E L}$ approaching zero. Figure 5 shows the resulting distribution of residuals, which has been fitted with a gaussian function. The width of the fitted gaussian peak is $14.6 \mu \mathrm{m}$, which is close to the expected value given by the strip pitch divided by $\sqrt{ } 12(14.4 \mu \mathrm{m})$.

\subsection{Spatial resolution with charge sharing included}

To investigate the effect of charge sharing on the detector's spatial resolution, detector hits were identified in terms of strip clusters. Initially the number of strips participating in the cluster (the cluster size) was allowed to fall within the range of 1 (a single strip only) to 5 strips (as an arbitrary upper limit) and the cluster threshold limit for each participating strip was varied from $0.5 \sigma$ to $5.0 \sigma$.

To determine the optimum value for the cluster threshold limit, residual distributions were derived for a number of threshold values, using $X_{D E T}$ determined by a weighted average of the strip pulse heights within the cluster (the centre of gravity method, see for example [11]), such that

$$
X_{D E T}=\frac{\sum_{i=1}^{n} P H_{i} x_{i}}{\sum_{i=1}^{n} P H_{i}}
$$


where $x_{i}$ is the position of centre of the $i$ th strip in the cluster.

The width of the resulting residuals distribution was found to reach a minimum when the threshold value was in the range $2.0 \sigma$ to $3.0 \sigma$. With a threshold value below $2.0 \sigma$, the average number of strips per cluster became artificially large and the width of the residual distribution rose accordingly.

The overall spatial resolution of the detector at different bias voltages was then determined using a fixed cluster threshold value of $2.0 \sigma$, plus at least one strip per cluster exceeding $5 \sigma$. At each bias voltage two separate residual distributions were constructed from (a) events with single strip hits only and (b) events with cluster sizes of 2 or more strips. The total residual distribution at each voltage was constructed from the sum of the two distributions. For those events containing clusters, the track position $X_{D E T}$ was determined from the $\eta$ distribution (see below). For clusters of size greater than 2 strips, the $\eta$ parameterisation was applied to the two adjoining strips which contained the largest combined pulse height.

For 2 strip clusters, it is convenient to describe the charge division between the two strips in terms of a parameter $\eta$, such that

$$
\eta=\frac{P H_{R}}{P H_{L}+P H_{R}}
$$

where $P H_{L}, P H_{R}$ are the signals from the strips on the left and right strip respectively. The resulting differential distribution $d N / d \eta$ normally has peaks at low $\eta$ and at high $\eta$, corresponding to events where the majority of the total charge is collected by the left or right strip respectively. In a simple strip detector configuration (ie. without intermediate strips) the distribution falls to a minimum at $\eta \approx 0.5$, corresponding to events which are incident mid way between the two strips and sharing their charge equally between them.

Using this parameterisation, the particle position $X_{D E T}$ is given by

$$
X_{D E T}=X_{L}+f(\eta) \times p
$$

where $X_{L}$ is the position of the centre of the left hand strip, $p$ is the detector strip pitch and $f(\eta)$ is an arbitrary monotonic function with $f(0)=0$ and $f(1)=1$. In the simplest case $f(\eta)$ is assumed to be linear, such that $f(\eta)=\eta$, which is the two-strip case of the more general centre of gravity position finding algorithm.

Figure 6 shows the $\eta$ distribution which was obtained for two strip clusters from the GaAs detector at a bias of $180 \mathrm{~V}$. Several aspects of the charge sharing effects in the detector can be identified from this distribution:

1. The fraction of total events with approximately equal charge division (in the $\eta$ range 0.4-0.6) is approximately $12 \%$, indicating less than ideal charge division in the inter-strip region.

2. There is a clear asymmetry between the distributions at low and high $\eta$. For example, the number of events in the $\eta$ range $0-0.3$ is $58 \%$ and the number between $0.7-1.0$ is $19 \%$.

The low proportion of total events at $\eta$ values close to 0.5 is similar to that observed in silicon strip detectors [12] and is largely related to the width of the charge diffusion cloud. In typical microstrip detectors fabricated from high resistivity silicon (eg. with a width of $300 \mu \mathrm{m}$ and a resistivity of a few $\mathrm{k} \Omega$ $\mathrm{cm})$ the diffusion cloud is of the order $5-10 \mu \mathrm{m}$ and so is considerably smaller than the strip pitch. The 
width of the diffusion cloud determines over how much of the interstrip region effective (ie. approximately equal) charge division occurs, so that as the ratio of the diffusion width over the strip pitch decreases there is a corresponding shift in the $\eta$ distribution of events out of the $\eta \approx 0.5$ region and into the peaks at low and high $\eta[13]$.

In the case of semi-insulating GaAs strip detectors, an understanding of the expected charge division is further complicated by the effect of charge trapping, whereby the charge released along the particle's track may not reach the surface contacts but instead be trapped within the detector volume. In this case the charges collected by the surface strip electrodes will be induced by the moving charge up to the time when the charge is trapped and the resulting distribution between strips will be determined by a combination of the strip geometry, the detector thickness and the mean position in the detector where the trapping occurs. For charges stopped by trapping close to the strip surface, the induced charge will be spread across several strips, and the measured cluster size will appear broader than that expected purely from diffusion.

The severity of charge trapping, and the resulting effect on the spatial resolution, are strongly dependent both on the material type and on the bias polarity of the readout strips. In this detector the mean drift lengths for electrons and holes are estimated to be of the order of $50 \mu \mathrm{m}$ and $350 \mu \mathrm{m}$ respectively [10]. Since the detector was biased to collect holes from the strip surface, the contribution to the total signal from the charge flowing towards the strips was due to the hole current, which was minimally affected by trapping.

However the observed asymmetry in the $\eta$ distribution is not expected for a detector arranged orthogonally to the incident beam. In this setup the detector was estimated to be orthogonal to the beam within a tolerance of less than $\pm 1^{\circ}$. The observed asymmetry in the eta distribution is consistent with electronic cross-talk between adjacent strips, caused by non-optimised adjustment of the SIROCCO sampling time. Accurate digitisation of the serial analogue levels which are input to the SIROCCO requires that the conversion should not occur until each analogue level has settled to its required value. Further measurements are being carried out at Sheffield using a ${ }^{90} \mathrm{Sr}$ source to investigate the sensitivity of the eta distribution to small adjustments in the SIROCCO sampling timing.

Figure 7 shows the resulting distribution of residuals obtained at $180 \mathrm{~V}$ from all events, categorised as either single strip or 2 strip cluster events (12\% and $88 \%$ of all events respectively). A gaussian function is shown fitted to the data and describes it well. The fitted gaussian has a width $\sigma=10.6 \mu \mathrm{m}$ which, after correction for the track resolution, gives a spatial resolution for the GaAs detector of $10.4 \mu \mathrm{m}$. A slight excess of events is visible on the left side of the peak, due to non-linearities in the charge sharing which are ignored by the use of the linear $f(\eta)=\eta$ position finding function.

Figure 8 shows the variation in spatial resolution obtained over bias values from $120 \mathrm{~V}$ to $180 \mathrm{~V}$. The resolution degrades from $10.4 \mu \mathrm{m}$ at $180 \mathrm{~V}$ bias to $11.2 \mu \mathrm{m}$ at $120 \mathrm{~V}$, due primarily to a corresponding drop in the proportion of 2 strip cluster events from $88 \%$ at $180 \mathrm{~V}$ to $70 \%$ at $120 \mathrm{~V}$.

\section{Conclusion}

The pulse height response and spatial resolution of the GaAs microstrip detector have been successfully demonstrated. The use of full analogue readout using VA2 electronics provided pulse height information from all the detector strips, which enabled cluster effects and charge sharing to be investigated.

The effect of charge sharing between pairs of strips has been clearly demonstrated, and appears to provide a substantial improvement in the detector's spatial resolution despite only using a simple linear position 
finding algorithm. Further laboratory tests and detector simulations will be carried out to investigate the cause of the asymmetric $\eta$ distribution.

For the next generation of GaAs detectors, improvements made to the contacts have been shown to allow operation at or close to 'full' bias, corresponding to the active high field region penetrating through the whole volume of the detector. Such devices have already been demonstrated to operate with charge collection efficiencies in excess of $80 \%$. These devices are currently undergoing beam tests, using fast prototype LHC readout electronics with shaping times of the order of 50ns.

\section{Acknowledgements}

The measurements reported here were made possible by the efforts of many people who participated in the H8 test-beam during August 1995. Particular thanks are due to Peter Weilhammer's group at CERN for supplying the VA2 readout electronics, to Ogmundur Runolfsson for bonding the detector modules, and to colleagues within the GaAs RD8 collaboration, particularly Alex Chilingarov and Saverio D'auria. Funding for SM was provided by the Greek Ministry of National Economy through the NATO fellowship programme, and for SW through a CASE studentship from EEV Ltd. This work was partly funded by the UK Particle Physics and Astronomy Research Council (PPARC).

\section{References}

[1] Status Report of the RD-8 Collaboration 1995, CERN/LHCC 95-97.

[2] D.S. McGregor and J.E. Kammeraad, in Semiconductors for Room Temperature Nuclear Detector Applications, edited by T.E. Schlesinger and R.B. James, in Semiconductors and Semimetals, Academic Press, San Diego, 43 (1995) 383.

[3] The GaAs detectors were fabricated by EEV Ltd, Chelmsford UK.

[4] S. Manolopoulos et al., submitted to Nuclear Instruments and Methods in Physics Research as the Proceedings of 3rd International. Workshop on GaAs and Related Compounds, San Miniato Italy, 1995.

[5] The resistor array was produced for the University of Sheffield by Dynamic Microcircuits Ltd, a division of British Aerospace, Filton, Bristol UK.

[6] The capacitor chip was designed by the CERN L3 collaboration.

[7] Originating at CERN and Oslo as the 'Viking' readout chip, the low noise 'VA2' version was manufactured by Integrated Detectors and Electronics AS (IDEAS), Oslo Norway.

[8] Originally supplied to CERN by LEPSI, Strasbourg. A commercial variant is now available from C.A.E.N. as the V550.

[9] J. Beringer et al., submitted to Nuclear Instruments and Methods in Physics Reseach as the Proceedings of the 2nd International Symposium on the Development and Application of Semiconductor Tracking Detectors, Hiroshima 1995.

[10] P.J. Sellin et al., IEEE Transactions on Nuclear Science 42/4 (1995) 247-253

[11] R. Turchetta, Nuclear Instruments and Methods in Physics Research, A335 (1993) 44-58.

[12] E. Belau et al., Nuclear Instruments and Methods, 214 (1983) 253-260.

[13] A description of the influence of diffusion width on the non-linear charge division in strip detectors can be found in Instrumentation in High Energy Physics, editor F. Sauli, volume 9 in Advanced Series on Directions in High Energy Physics, World Scientific, Singapore 1992. 


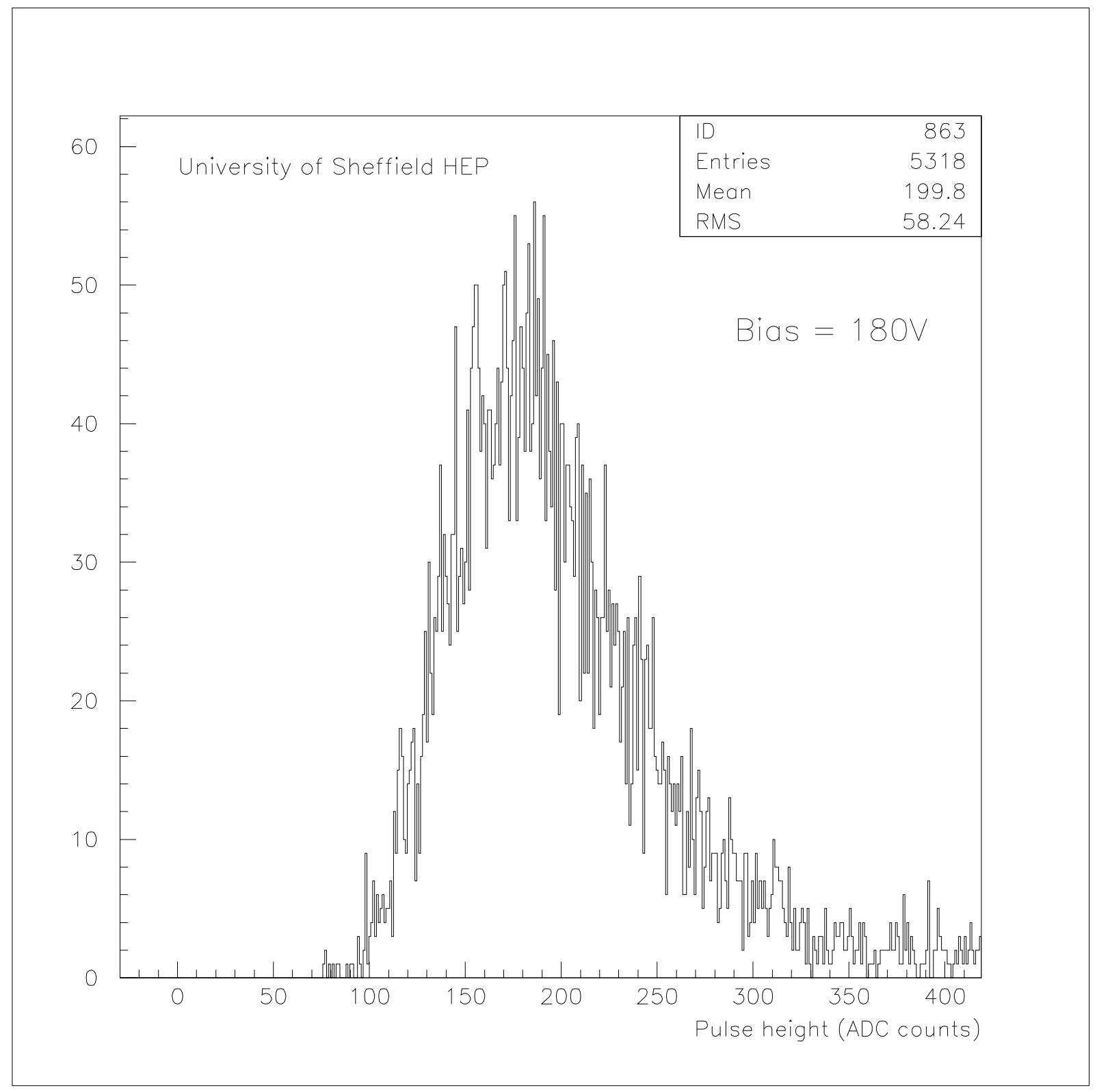

Figure 1: GaAs detector pulse height spectrum, at a detector bias of 180V. 


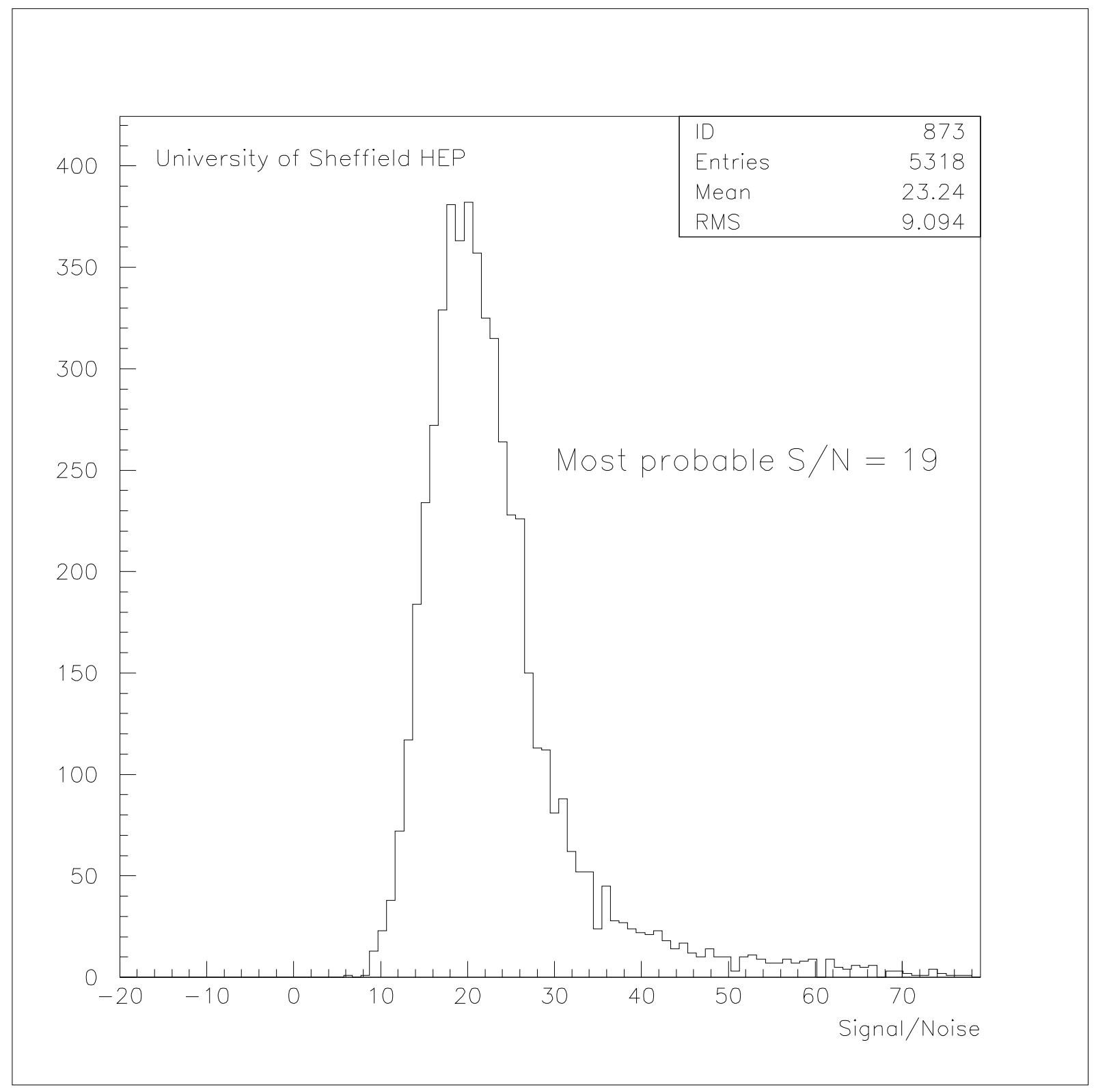

Figure 2: The pulse height data from figure 1 plotted in units of signal over noise. The most probable signal over noise value, calculated by fitting a gaussian-convoluted Landau distribution, is approximately 19. 


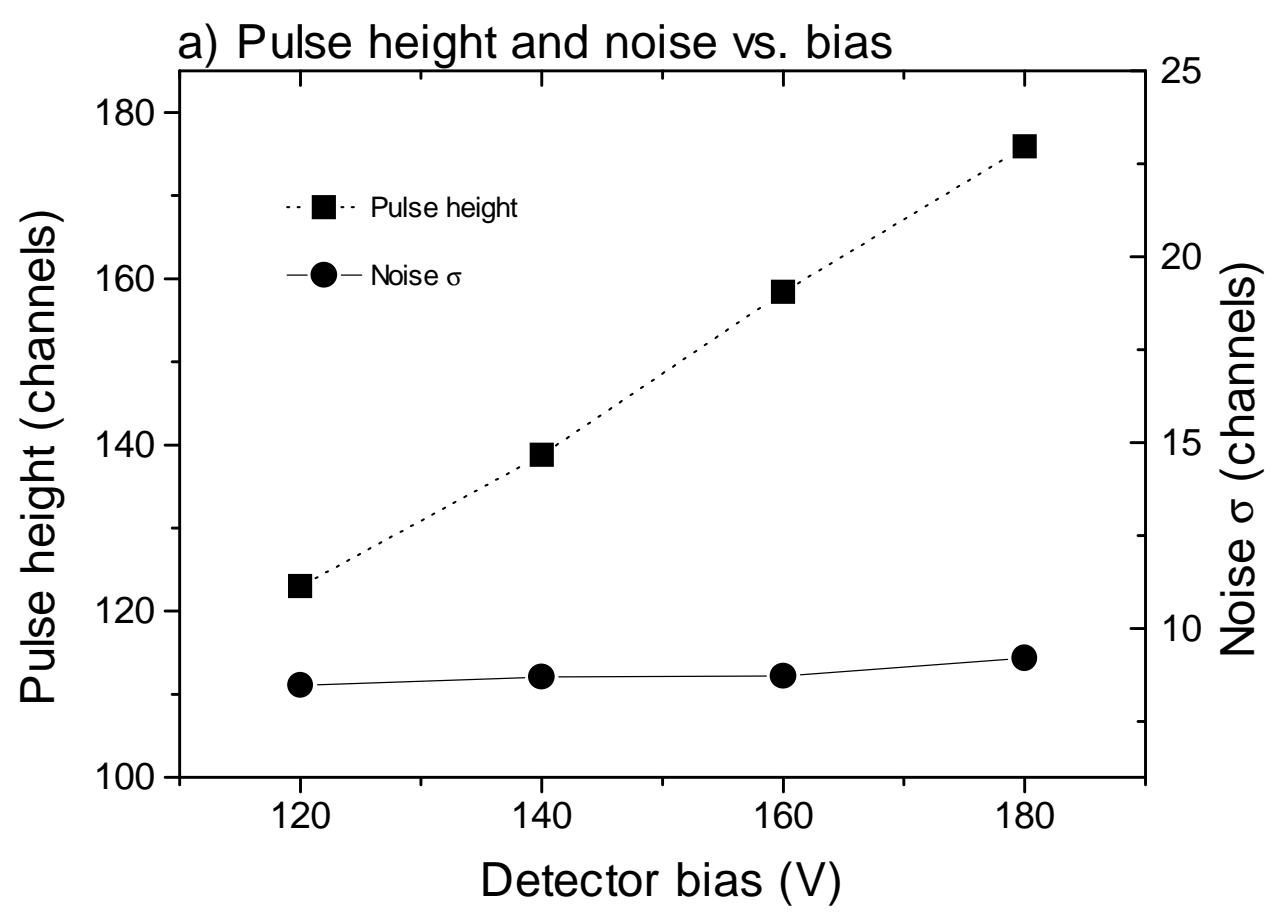

b) Pulse height and signal/noise vs. bias

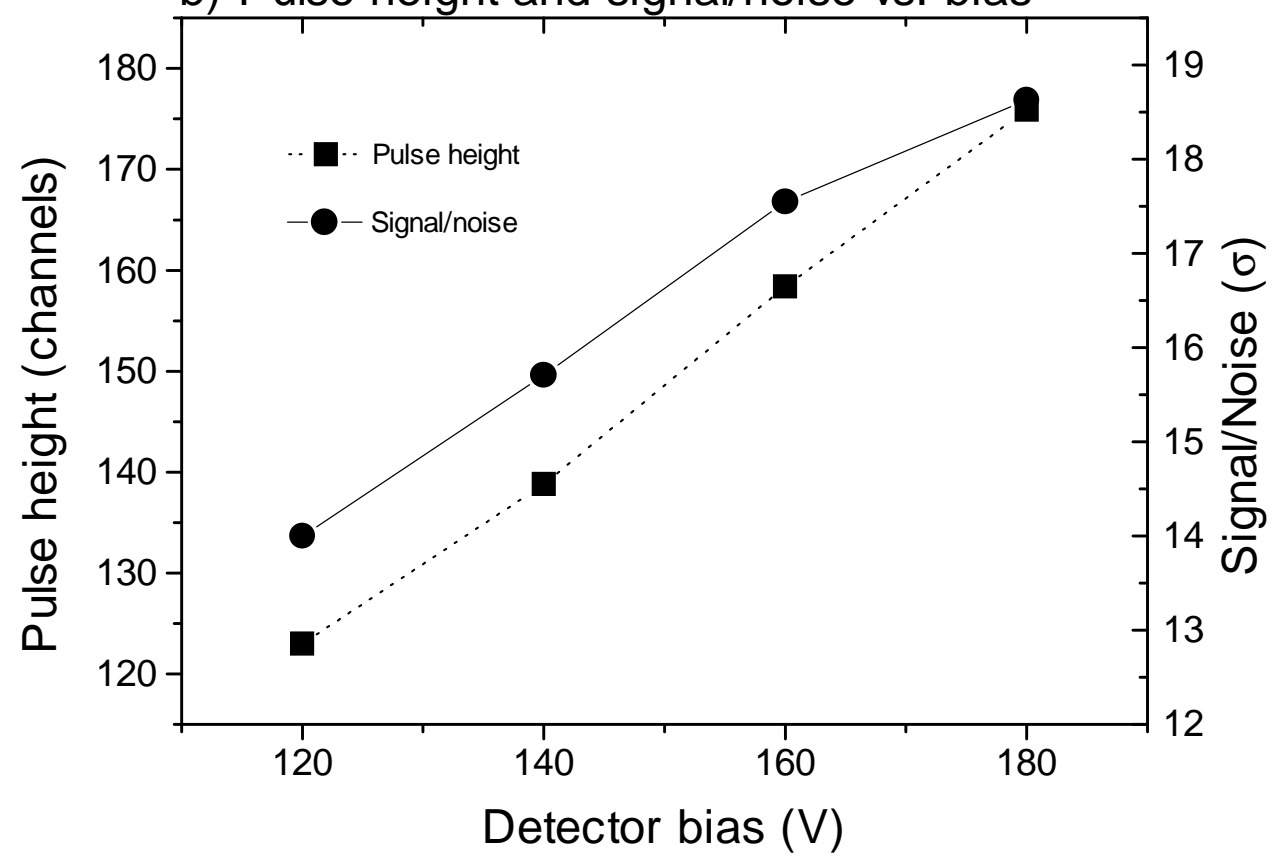

Figure 3: Plots of a) the most probable value of the pulse height and the average noise per strip, and b) the most probable values of the pulse height and signal to noise ratio, as a function of detector bias voltage. 


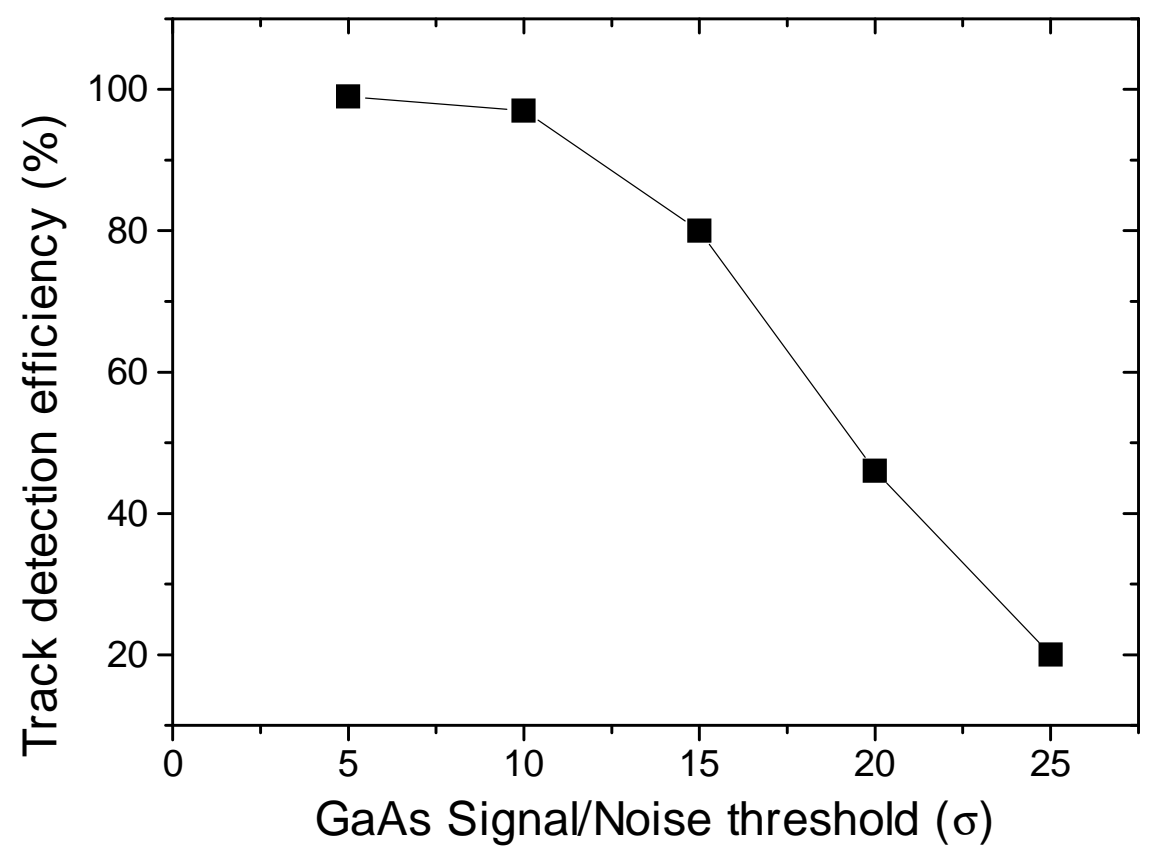

Figure 4: Track detection efficiency in the GaAs detector, plotted as a function of single strip signal/noise threshold. 


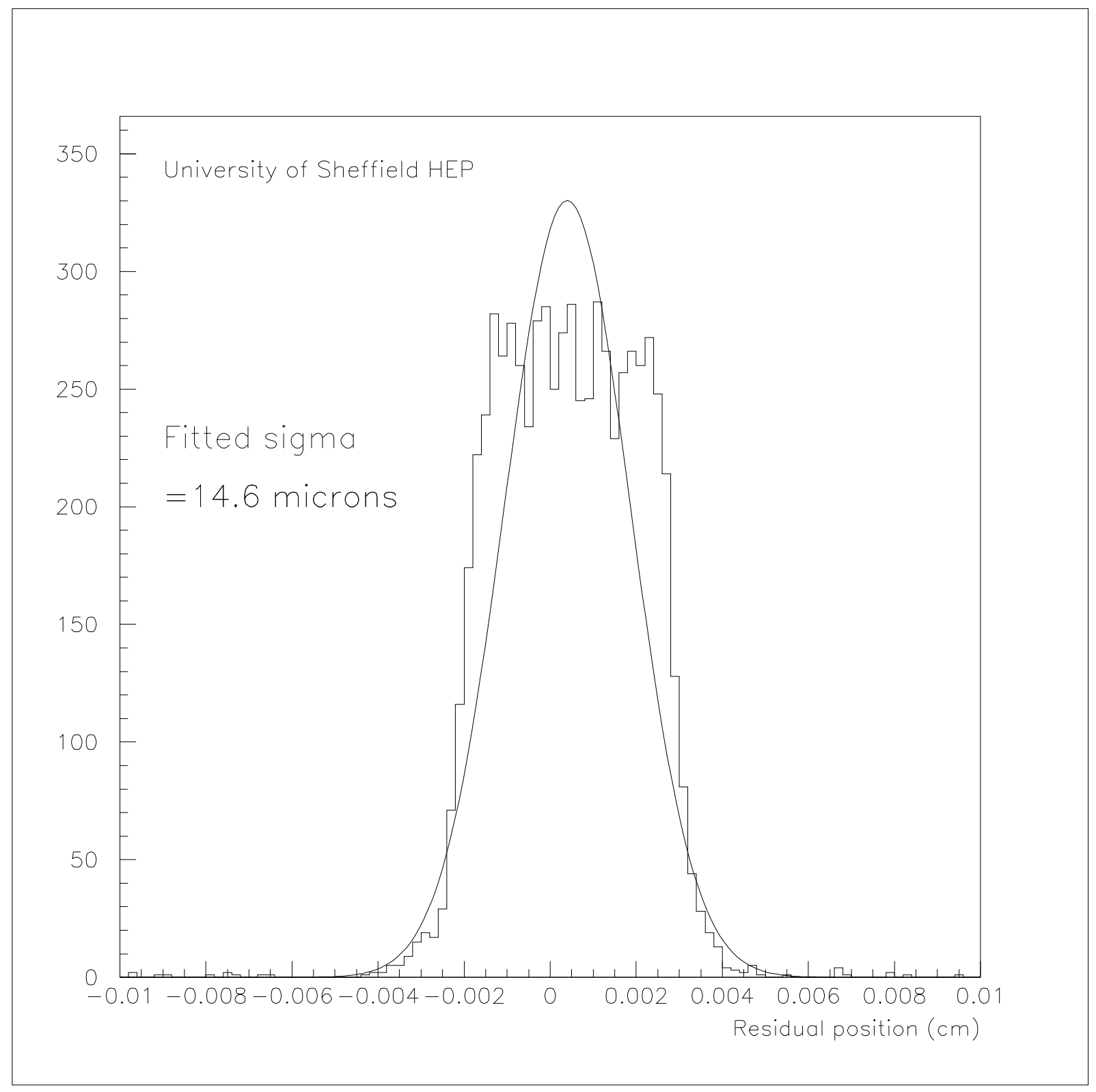

Figure 5: Residuals distribution without charge sharing; the hit for each event is defined as the single strip with the largest pulse height (and with $\mathrm{PH}>5 \sigma$ ). 


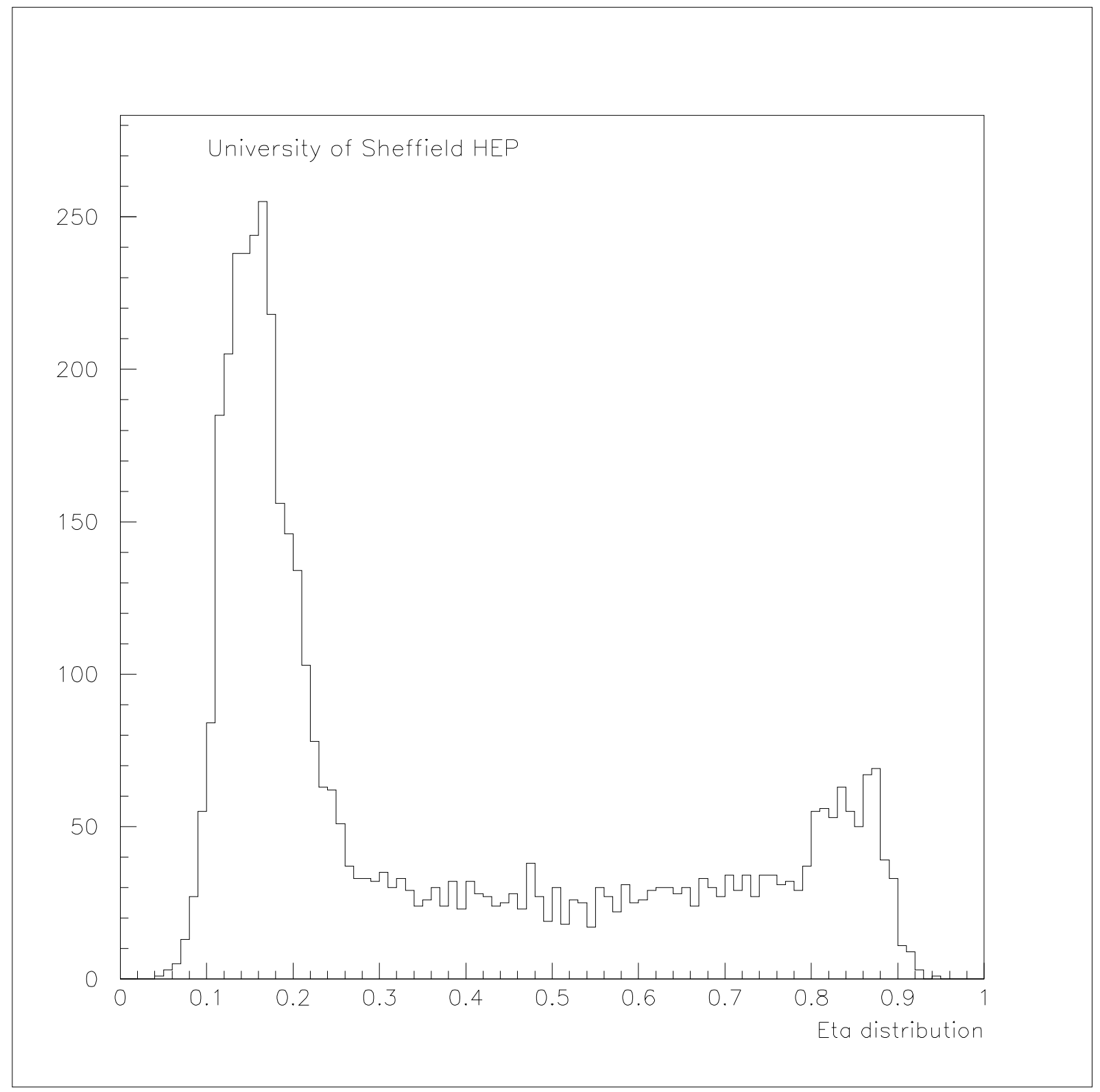

Figure 6: $\eta$ distribution for 2 strip clusters at $180 \mathrm{~V}$ bias. The unexpected asymmetry in the distribution is consistent with electronic cross-talk between strips (see text). 


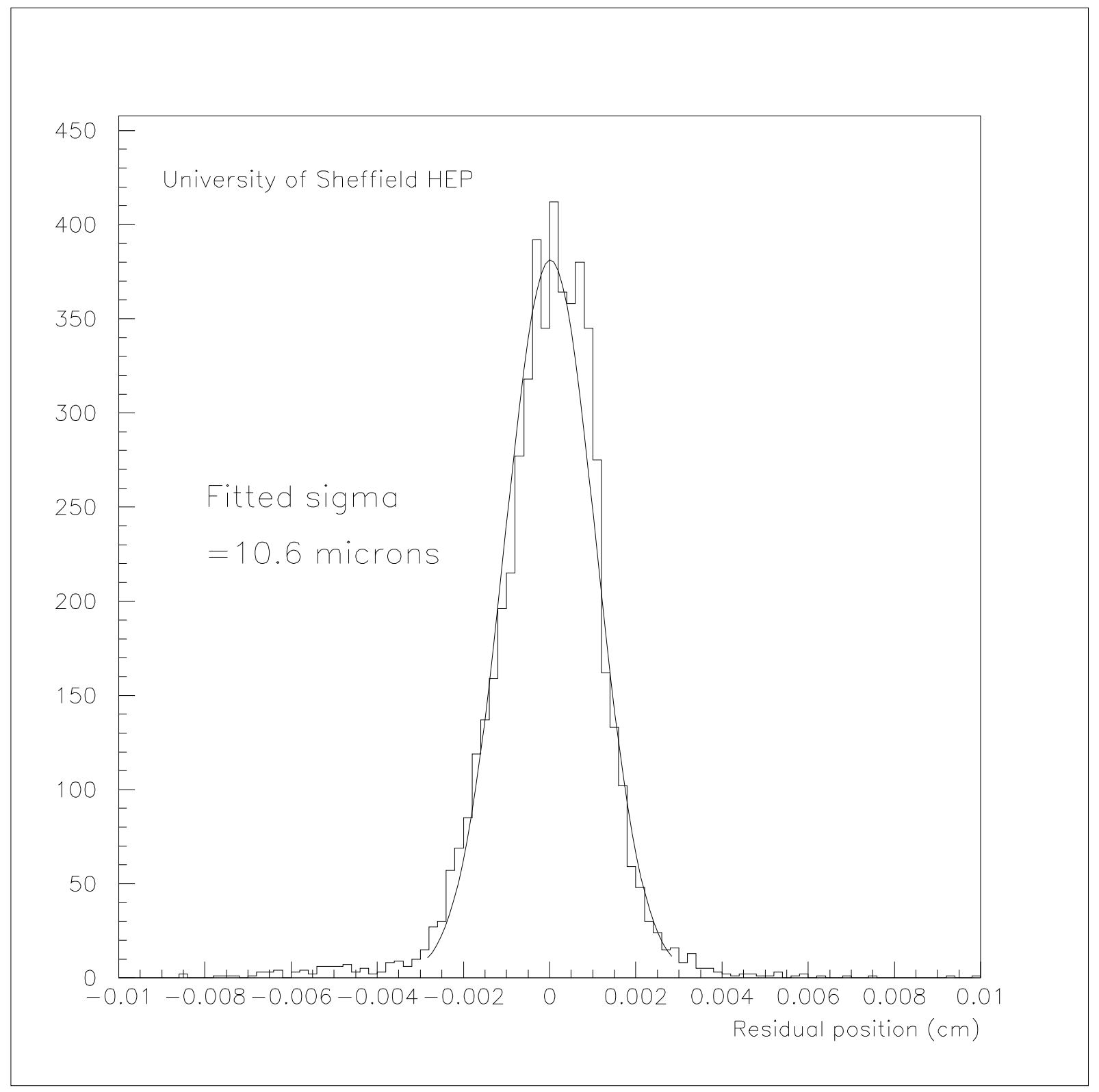

Figure 7: Residuals distribution with charge sharing, for all events at $180 \mathrm{~V}$ bias. After correcting for the telescope resolution, the overall spatial resolution was $10.4 \mu \mathrm{m}$. 


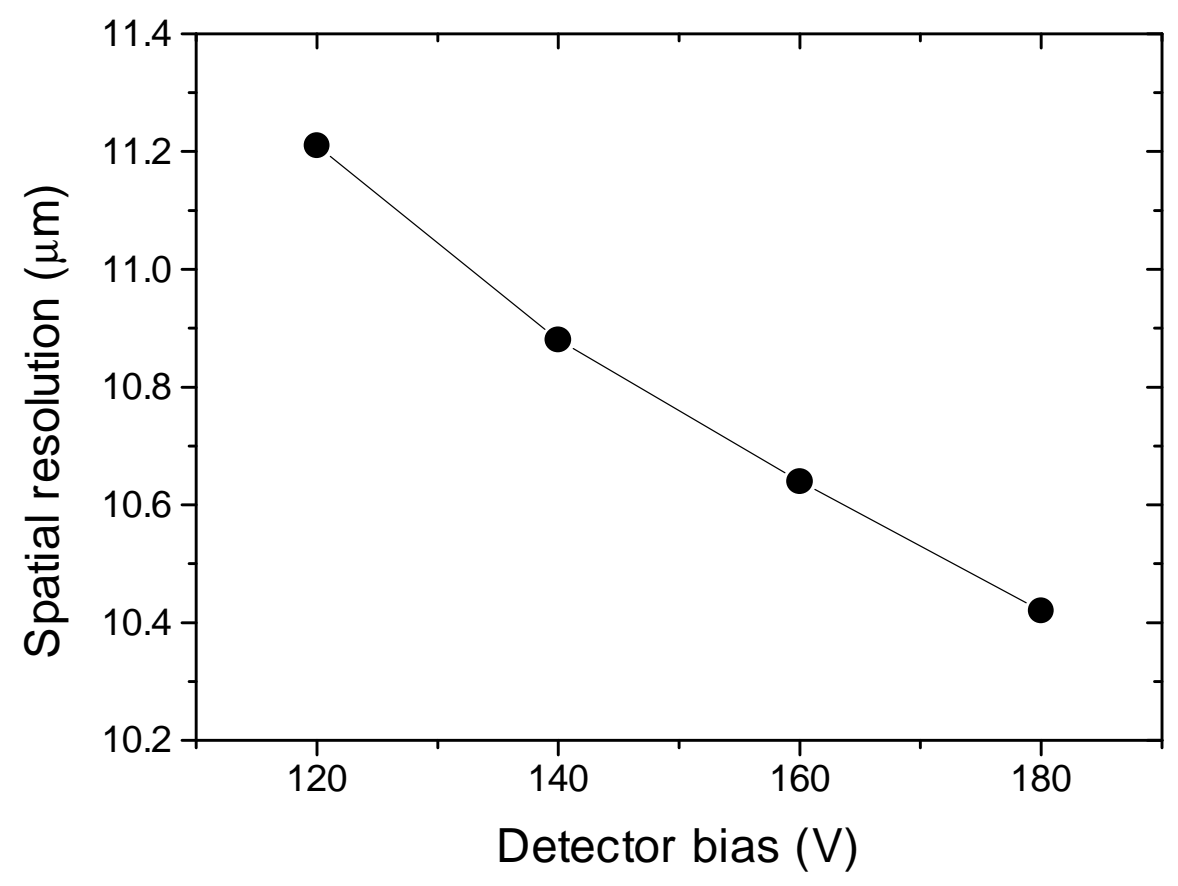

Figure 8: Overall detector resolution using charge sharing, obtained from all events as a function of detector bias. 